\title{
Probing the 3-D matter distribution at $z \sim 2$ with QSO multiple lines of sight $\dagger$
}

\author{
S. Cristiani ${ }^{1}$, V. D'Odorico ${ }^{1}$, F. Saitta ${ }^{2}$, M. Viel $^{3}$, S. Bianchi $^{4}$, \\ B. Boyle ${ }^{5}$, S. Lopez ${ }^{6}$, J. Maza ${ }^{6}$ and P. Outram ${ }^{7}$ \\ ${ }^{1}$ INAF - Osservatorio Astronomico di Trieste, via G.B. Tiepolo, 11 I-34131 Trieste, Italy, \\ email: cristiani@ts.astro.it, dodorico@ts.astro.it \\ ${ }^{2}$ Dipartimento di Astronomia, Università degli Studi di Trieste, via G.B. Tiepolo, 11 \\ I-34131 Trieste, Italy \\ ${ }^{3}$ Institute of Astronomy, Madingley Road, Cambridge CB3 0HA \\ ${ }^{4}$ INAF-Istituto di Radioastronomia/Sez. Firenze, Largo Enrico Fermi, 5, \\ I-50125 Firenze, Italy \\ ${ }^{5}$ Australia Telescope National Facility, PO Box 76, Epping NSW 1710, Australia \\ ${ }^{6}$ Departamento de Astronomia, Universidad de Chile, Casilla 36-D, Santiago, Chile \\ ${ }^{7}$ Department of Physics, University of Durham, South Road, Durham, DH1 3LE
}

\begin{abstract}
We investigate the 3-D matter distribution at $z \sim 2$ with high resolution $(R \sim$ $40,000)$ spectra of QSO pairs and groups obtained with the UVES spectrograph at ESO VLT. Our sample is unique for the number density of objects and the variety of separations, between $\sim 0.5$ and 7 proper Mpc $\dagger$. We compute the real space cross-correlation function of the Lyman- $\alpha$ forest transmitted fluxes. There is a significant clustering signal up to $\sim 2$ proper Mpc, which is still present when absorption lines with high column density $(\log N \geqslant 13.8)$ are excluded.
\end{abstract}

\section{Introduction}

The Ly- $\alpha$ forest absorbers detected at $z>1.5$ in high resolution QSO spectra outnumber any other population of objects observable from the ground at those redshifts. They originate, as shown by hydro-simulations (e.g. Cen et al. 1994), in density fluctuations of the intergalactic medium (IGM), i.e. in the web of filamentary structures connecting the densest peaks, and are reliable tracers of the baryon density field as well as of the underlying dark matter distribution.

From the study of absorption spectra along single lines of sight (LOSs) to distant QSOs it has been possible to determine the shape and amplitude of the power spectrum of the spatial distribution of dark matter (e.g. Kim et al. 2004, Viel, this conference), and gain important information on the baryon density of the Universe (Rauch et al. 1997) and on the physical state of the IGM (Schaye et al. 2000).

Our present analysis introduces the use of adjacent LOSs to probe the actual 3-D distribution of matter in the Universe and provide estimates of the size/correlation of the absorbing structures. Hydrodynamical (Charlton et al. 1997) and analytical (Viel et al. 2002) simulations have shown the advantages in using multiple LOSs with respect to single ones, in particular, to decrease the effects of inaccuracies in the continuum fitting.

$\dagger$ Based on observations collected at the European Southern Observatory, Chile $\dagger$ We adopt a $\Lambda$ CDM cosmology with $\Omega_{m}=0.3, \Omega_{\Lambda}=0.7$, and $h=0.72$. 


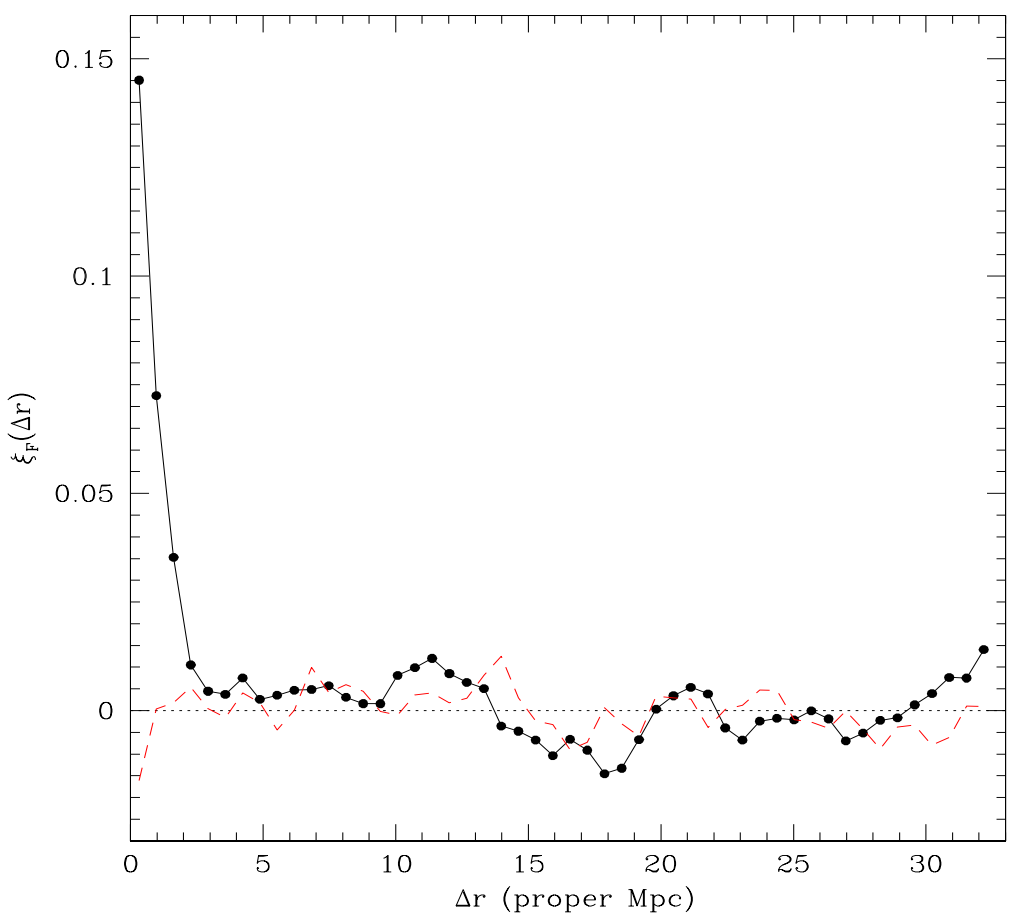

Figure 1. Correlation function of the transmitted flux in real space for our sample of pairs and groups of QSOs. The dashed line is the result obtained for the uncorrelated control sample (see text).

\section{Our sample of QSOs}

Two major breakthroughs have dramatically improved the exploitation of the potential offered by QSO multiple LOSs: the 2dF QSO Redshift Survey (2QZ, Croom et al. 2004), whose complete spectroscopic catalogue contains more than $\sim 23,000$ QSOs, approximately 50 times more than the previous largest QSO survey to a similar depth $(B<21)$ and the UVES spectrograph at the ESO VLT telescope which has a remarkable efficiency especially in the extreme UV.

We have searched the $2 \mathrm{dF}$ and other QSO databases for the best groups with apparent magnitude $B \leqslant 20$ and $z>1.8$ and carried on a great observational effort to collect UVES spectra of the selected QSOs. At the moment, we have observed one QSO pair, one triplet, and one sextet of QSOs with a resolution $R \sim 40,000$ and signal-to-noise ratio in the Lyman- $\alpha$ forest larger than 3. With the addition of two more UVES QSO pairs from our archive, we total 20 different baselines with proper spatial separations between $\sim 0.5$ and $7 \mathrm{Mpc}$, a unique sample both for the number density and the variety of LOS separations investigated.

\section{The transmitted flux cross-correlation function}

We select in each normalised spectrum the region between the Lyman- $\beta$ emission (or the minimum observed wavelength, when the Lyman- $\beta$ falls outside the spectrum) and $5000 \mathrm{~km} \mathrm{~s}^{-1}$ from the Lyman- $\alpha$ emission (to avoid proximity effect) and compute the cross-correlation function of the transmitted flux between adjacent LOSs. The following 
generalisation of the formula for the density field (e.g. Peebles 1980) has been adopted:

$$
\xi_{F}(\Delta r)=\frac{<(F(x)-<F>)\left(F_{0}(x+\Delta r)-<F_{0}>\right)>}{\sqrt{<(F(x)-<F>)^{2}><\left(F_{0}(x+\Delta r)-<F_{0}>\right)^{2}>}}
$$

where $F$ and $F_{0}$ are the transmitted fluxes in two adjacent LOSs and $\Delta r$ is the proper spatial separation between two pixels in different LOSs. The cross-correlation function is estimated over all the considered pixels of all the QSO pairs in the sample. To check for the effects of systematics we also computed the same cross-correlation function on a control sample, obtained by substituting in turn one of the QSO spectra in each baseline with an 'uncorrelated' one, roughly at the same redshift, but far away on the sky, picked up in our database of high-resolution, high-S/N QSO spectra. The result is shown in Fig. 1. A correlation signal is present up to proper separations of $\sim 2 \mathrm{Mpc}$ indicating a large coherence length of the absorbers. This length is consistent with the size of Lyman$\alpha$ absorbers we found in D'Odorico et al. (1998) with the line analysis of coincidences and anti-coincidences.

In order to verify the relative contribution of the stronger and weaker absorption lines to the clustering signal we have selected the pixels with $F>0.2$ corresponding to $\log N(\mathrm{HI})<13.7-13.8$ and recomputed $\xi_{F}(\Delta r)$. Although significantly decreased (by a factor $\sim 2$ ) a clustering signal is still clearly present. This is an indication that also far from the most over-dense regions, in the 'true' IGM, matter is still clustered.

A comparison with hydrodynamical simulations (see for a description, Viel et al. 2004) shows a substantial agreement between the predicted and observed signal.

\section{References}

Cen, R., Miralda-Escudé, J., Ostriker, J. P., Rauch, M., 1994, ApJ, 437, L83

Charlton, J. C., Anninos, P., Zhang, Y., Norman, M. L., 1997, ApJ, 485, 26

Croom, S. M., Smith, R. J., Boyle, B. J., Shanks, T., Miller, L., Outram, P. J., Loaring, N. S., 2004, MNRAS, 349, 1397

D’Odorico, V., Cristiani, S., D’Odorico, S., Fontana, A., Giallongo, E., Shaver, P., 1998, A\&A, 339,678

Kim, T.-S., Viel, M., Haehnelt, M., Carswell, R. F., Cristiani, S., 2004, MNRAS, 347, 355

Peebles, P. J. E., 1980, in The Large-Scale Structure of the Universe. Princeton University Press

Rauch, M., Miralda-Escude, J., Sargent, W. L. W., Barlow, T. A., Weinberg, D. H., Hernquist, L., Katz, N., Cen, R., Ostriker, J. P., 1997, ApJ, 489, 7

Schaye, J., Theuns, T., Rauch, M., Efstathiou, G., Sargent, W. L. W., 2000, MNRAS, 318, 817

Viel, M., Haehnelt, M. G., Springel, V., 2004, MNRAS, 354, 684

Viel, M., Matarrese, S., Mo, H. J., Haehnelt, M., Theuns, T., 2002, MNRAS, 329, 848 